NASA Technical Memorandum 100433

\title{
Flight Tests of External Modifications Used to Reduce Blunt Base Drag
}

\section{Sheryll Goecke Powers}

(BASA-TH-100433) FIIGHT TESIS OF EXTERAAL MCDIPICATICNS TSED TO BEDUCE ELUAT BASE DAAG (BASA) $17 \mathrm{~F}$
N88-2C279

Unclas

0136045

June 1988

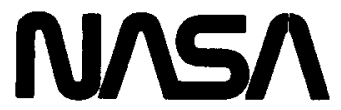

National Aeronautics and

Space Administration 
NASA Technical Memorandum 100433

\section{Flight Tests of External Modifications Used to Reduce Blunt Base Drag}

Sheryll Goecke Powers

Ames Research Center, Dryden Flight Research Facility, Edwards, California

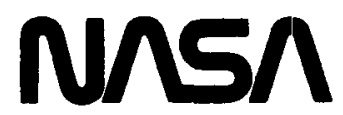

National Aeronautics and

Space Administration

Ames Research Center

Dryden Flight Research Facility

Edwards, California 93523-5000 


\title{
FLIGHT TESTS OF EXTERNAL MODIFCATIONS USED TO REDUCE BLUNT BASE DRAG
}

\author{
Sheryll Goecke Powers* \\ Ames Research Center \\ Dryden Flight Research Facility \\ Edwards, California
}

\begin{abstract}
The effectiveriess of a trailing disk (the trapped vortex concept) in reducing the blunt base drag of an 8-in. diameter body of revolution was studied from measurements made both in fight and in full-scale wind-tunnel tests. The experiment demonstrated the significant base drag reduction capability of the trailing disk to Mach 0.93. The maximum base drag reduction obtained from a cavity tested on the flight body of revolution was not significant. The effectiveness of a splitter plate and a vented-wall cavity in reducing the base drag of a quasi-two-dimensional fuselage closure was studied from base pressure measurements made in flight. The fuselage closure was between the two engines of the F-111 airplane, and, thus, the base pressures were in the presence of jet engine exhaust. For Mach numbers from 1.10 to 1.51, significant base drag reduction was provided by the vented-wall cavity configuration. The splitter plate was not considered effective in reducing base drag at any Mach number tested.
\end{abstract}

\section{Nomenclature}

\begin{tabular}{|c|c|}
\hline$A_{B}$ & BOR base area, $\mathrm{ft}^{2}$ \\
\hline$A_{F}$ & fuselage closure base area, $\mathrm{ft}^{2}$ \\
\hline$A_{v B}$ & slot area for BOR vented-wall cavity, $\mathrm{ft}^{2}$ \\
\hline$A_{\mathrm{u} F}$ & $\begin{array}{l}\text { slot area for fuselage closure vented-wall } \\
\text { cavity, } \mathrm{ft}^{2}\end{array}$ \\
\hline BOR & body of revolution \\
\hline$b$ & $C_{p-\text { axis intercept }}$ \\
\hline$C_{p}$ & $\left(p_{i}-p\right) / 0.7 M^{2} p$ \\
\hline$C_{p}(R)$ & linear pressure coefficient function, $m R+b$ \\
\hline$C_{D}$ & total BOR drag coefficient \\
\hline$C_{D_{b}}$ & base drag coefficient \\
\hline$D$ & base diameter, in. \\
\hline$D B$ & base drag, lb \\
\hline$D S$ & drag for a given surface, lb \\
\hline$d$ & depth of cavity, in. \\
\hline FLTD & flight trailing disk \\
\hline$L$ & length of body, in. \\
\hline
\end{tabular}

\footnotetext{
-Aerospace engineer, AlAA member.
}

\begin{tabular}{|c|c|}
\hline$M$ & local reference Mach number \\
\hline$M_{\infty}$ & free-stream Mach number \\
\hline$m$ & slope, in..$^{-1}$ \\
\hline$p$ & local reference pressure, $\mathrm{lb} / \mathrm{ft}^{2}$ \\
\hline$p_{b}$ & base pressure \\
\hline$p_{i}$ & pressure at a given orifice, $1 \mathrm{~b} / \mathrm{ft}^{2}$ \\
\hline$p_{T}$ & turbine discharge total pressure, $\mathrm{lb} / \mathrm{ft}^{2}$ \\
\hline$p_{\infty}$ & free-stream pressure, $\mathrm{lb} / \mathrm{ft}^{2}$ \\
\hline$q$ & local dynamic pressure, $0.7 M^{2} p$ \\
\hline$R$ & radius, in. \\
\hline$R B$ & radius of base, in. \\
\hline$r_{\max }$ & $\begin{array}{l}\text { radius to edge of BOR base or to edge of } \\
\text { trailing disk, in. }\end{array}$ \\
\hline$r_{\min }$ & $\begin{array}{l}\text { minimum radius value, in.; } 0 \text { in. if stem not } \\
\text { present; } 0.50 \text { in. if stem present }\end{array}$ \\
\hline TACT & transonic aircraft technology \\
\hline WTD & wind-tunnel trailing disk \\
\hline$x$ & $\begin{array}{l}\text { distance between base of BOR and upstream } \\
\text { surface of trailing disk, in. }\end{array}$ \\
\hline$\Delta C_{D}$ & $\begin{array}{l}\text { difference between } C_{D} \text { of trailing disk configuration } \\
\text { and } C_{D} \text { of blunt base configuration }\end{array}$ \\
\hline$\Delta C_{D_{b}}$ & $\begin{array}{l}\text { difference between } C_{D_{\mathrm{b}}} \text { of modified configuration } \\
\text { and } C_{D_{\mathrm{b}}} \text { of blunt base configuration }\end{array}$ \\
\hline$\alpha$ & angle of attack, deg \\
\hline$\theta$ & $\begin{array}{l}\text { angular orientation with respect to top dead center, } \\
\text { deg; } 0^{\circ} \text { at top, increasing in clockwise direction } \\
\text { when body is viewed from rear }\end{array}$ \\
\hline
\end{tabular}

\section{Summary}

The effectiveness of external modifications in reducing the in-flight base drag of a body of revolution and a quasi-twodimensional fuselage closure was studied. The body of revolution was the fin cap for the vertical fin of an F-111 aircraft. The fuselage closure was between the two engines of the F-111 airplane, and, thus, the base pressures were in the presence of jet engine exhaust. All data were analyzed using the blunt base as the reference condition. 
The effectiveness of a trailing disk (the trapped vortex concept) in reducing the base drag of the 8-in. diameter body of revolution was studied from measurements made both in flight and and in fullscale wind-tunnel tests. The experiment demonstrated the significant base drag reduction capability of the trailing disk to Mach 0.93. For the trailing disk data from the flight experiment, the maximum decrease in base drag coefficient ranged from 0.08 to 0.07 as Mach number increased from 0.70 to 0.93 . For the trailing disk data from the wind-tunnel experiment, the maximum decrease in base drag and total drag coefficients ranged from 0.08 to 0.05 as Mach number increased from 0.30 to 0.82 . The maximum base drag reduction obtained with a cavity tested in fight was not considered significant.

For the fuselage closure, the effectiveness of a splitter plate and a vented-wall cavity in reducing base drag was studied from base pressure measurements made in flight. For Mach numbers from 1.10 to 1.51 , significant base drag reduction was provided by the vented-wall cavity configuration. The increments ranged from 0.07 to 0.05 and resulted in base drag reductions of 27 and 24 percent at Mach numbers of 1.31 and 1.51 , respectively. The splitter plate was not considered effective in reducing base drag at any Mach number tested.

\section{Introduction}

Flight requirements can often result in aircraft configurations with high drag base regions such as a blunt base. Streamlined, axisymmetric shapes with blunt bases are commonly found on aircraft; sometimes as integral components such as fin caps, midwing tanks or wing-tip tanks; and sometimes as removable components such as missiles, external fuel tanks or other external stores. Quasitwo-dimensional blunt bases are often the result of propulsion configuration requirements or the result of aerodynamic stability requirements such as those that apply to hypersonic vehicles, for example. It is usually desirable, if possible, to reduce the base drag of these shapes and thereby reduce the total in-flight drag of the aircraft.

For streamlined axisymmetric shapes, vortex shedding from the blunt base can be the dominant drag component. Use of vortex control with three-dimensional shapes was discussed by Ringleb' ${ }^{\prime}$ and investigated by Migay $^{2}$ in the early 1960s. Incompressible (approximately 0.1 Mach number), turbulent flow wind-tunnel studies by $\mathrm{Mair}^{3}$ and Goodyer ${ }^{4}$ showed that for a blunt base body of revolution, a trailing disk (a disk mounted parallel to and slightly aft of the base plane) could establish a trapped toroidal vortex which favorably affected the way in which the flow closed around the base region. In effect, the trailing disk caused the flow to behave as though the base region ended in a beneficial boattail closure; and thus, the use of a trailing disk resulted in a substantial reduction in base drag. Incompressible, turbulent flow wind-tunnel studies by Mair ${ }^{5}$ showed that a cavity could also be successful in reducing the base drag of a three-dimensional body.

Two-dimensional studies have shown that interfering with the vortex formation in the wake can reduce the base drag component caused by vortex shedding. ${ }^{6}$ For example, incompressible, low
Reynolds number, wind-tunnel studies by Roshko ${ }^{7}$ and Bearman ${ }^{8}$ have shown that the effects of vortex formation and hence the base drag of a two-dimensional configuration could be reduced by placing a splitter plate in the wake. Both the eddy-shedding frequency and the location of the vortices were affected by the splitter plate. A quasi-two-dimensional flight study ${ }^{9}$ successfully demonstrated the effectiveness of the splitter plate, or vortex control concept, for Reynolds numbers near $10^{7}$ and Mach numbers to 0.90 . In another example, the two-dimensional wind-tunnel study of Nash and others ${ }^{10}$ showed that a cavity at the trailing edge of a blunt base shape could reduce the base drag for subsonic Mach numbers but not for supersonic Mach numbers.

An F-111 airplane used in the transonic aircraft technology (TACT) research flights ${ }^{11}$ at NASA Ames-Dryden provided an opportunity to study the effects of base modifications on a body of revolution and a quasi-two-dimensional shape. The fin cap (a body of revolution shape) at the top of the F-111 airplane's vertical fin was used to evaluate the effect of a trailing disk ${ }^{12}$ and of a cavity on the base drag. The 8-in. base diameter of the body of revolution was larger than the previous studies of Mair, ${ }^{3,5}$ and Goodyer. ${ }^{4}$ The Mach numbers and Reynolds numbers were also larger than those of the previous studies.

The quasi-two-dimensional fuselage closure of the F-111 airplane, unlike the bases of the previous two-dimensional and quasitwo-dimensional studies, was in the presence of jet exhaust. The fuselage closure of the F-111 airplane, between and behind the aircraft's two jet engines, was used to evaluate the effect of a splitter plate and a cavity on the base drag of a blunt base body in the presence of jet engine exhaust. ${ }^{13}$

The effects of the base modifications on the base pressures of the body of revolution and the fuselage closure are summarized.

\section{Description of Experiment}

A photograph of the F-111 TACT airplane in flight is shown in Fig. 1. The locations of the body of revolution (BOR) and fuselage closure are also shown. The BOR, shown again in Fig. 2(a), was the fin cap for the vertical fin which had a height of $8.9 \mathrm{ft}$. The vented-wall cavity base is installed. The trailing edge thickness of the vertical fin approached the thickness of the skin surface material. The precipitation static discharge probe and navigation light along the trailing edge of the vertical fin were present as shown in Fig. 2(a) during all flights for this study.

The peanut shape of the fuselage closure, the fuselage closure base, and the location of the fuselage closure base with respect to the engines is seen in Fig. 2(b). The fuel dump for the aircraft is near the bottom of the fuselage closure. The distance between the centerlines of the two engines, at the engine exit plane, is 60 in. The engine exit plane is approximately $18 \mathrm{in}$. forward of the base plane of the fuselage closure. The two engines are Pratt and Whitney (West Palm Beach, Florida) TF30-P-3 axial flow, twinspool turbofans. Further details about the aircraft and the propulsion system may be obtained from Painter and Caw ${ }^{11}$ and Cooper and others. ${ }^{14}$ Angles of attack and sideslip, free-stream impact and static pressures, and other airplane quantities were obtained from the airplane's calibrated noseboom system. 


\section{Flight Body of Revolution}

The 86.6-in.-long BOR has an ogive nose followed by a cylindrical centerbody and afterbody (Fig. 2(a)). The fineness ratio $L / D$ is 10.9. The base modifications for the BOR were investigated in two separate studies-the trailing disk study and the base cavity study. The base shapes investigated in the trailing disk study were the blunt base and the trailing disk (Fig. 3). The blunt base, as indicated by the name, provided an abrupt $90^{\circ}$ change in the surface contour. The trailing disk was connected from the center of the upstream disk surface to the center of the base of the BOR by a 1.0 -in. diameter stem. The dimensions for the trailing disk and stem (Fig. 3(b)) were determined from the data in Mair ${ }^{3}$ and Goodyer. ${ }^{4}$ The separation distance $x$ between the body base and the upstream disk surface (Fig. 4) could be adjusted.

The BOR base for the base cavity study differed from the previous base in that an extension protruded $0.92 \mathrm{in}$. aft of the base plane (dashed line in Fig. 5 indicates original base plane) of the BOR. The cavity shapes were slipped over the base extension; thus, the cavity bases fit onto the BOR without causing a step discontinuity in the external wetted surface. A 0.92 -in.-wide ring was used when a blunt base was required. The base shapes investigated were the extended blunt base, the solid- wall cavity, and the vented-wall cavity (Fig. 2(a)). Both the solid-wall and vented-wall cavities had the same ratio of depth (distance from base face to trailing edge of the cavity) to base diameter, $d / D$, of 0.35 . Cavity and slot dimensions are given in Fig. 5. The ratio of slot area to blunt base area $A_{v B} / A_{B}$ is 0.42 . The $d / D$ and $A_{v B} / A_{B}$ ratios used were determined from data in Mair. ${ }^{5}$

Pressures were measured both on the surface of the BOR and on the base surfaces. The angular orientation of the pressure orifice rows, $\theta$, is shown in Fig. 4. The orifice locations for the trailing disk study are found in Powers and others. ${ }^{12}$ The bases for the base cavity study had orifices along rows at four angular locations instead of two. All joints and openings on the body surface and in the base region were carefully sealed for each of the configurations to ensure that air leakage from inside the BOR did not exist. The body and base surface pressures were obtained using a 48-port multiplexing valve with a differential pressure transducer.

\section{Wind-Tunnel Body of Revolution}

A full-scale model of the BOR (Fig. 6) was tested in the NASA Langley high-speed 7 - by $10-\mathrm{ft}$ tunnel. ${ }^{15}$ The model was constructed from an aircraft loft drawing for the F-111 vertical fin cap; thus, differences between the flight and wind-tunnel BOR shapes are minimal. The only significant difference between the BOR configuration that was tested in flight and the one tested in the wind tunnel was the method of mounting (see Fig. 2(a) for flight mount and Fig. 6 for wind-tunnel mount). Details about the wind-tunnel BOR, the mounting strut, and pressure orifice locations are found in Powers and others. ${ }^{12}$

The base shapes investigated in the wind-tunnel study were the blun: base, the trailing disk flown in the flight study (FLTD), and the wind-tunnel trailing disk (WTD). The WTD was constructed to the dimensions of the FLTD but had a longer stem, enabling a wider range of separation distances to be studied.
The total BOR drag was obtained using force data from an electrical strain gage. Multiplexing valves with differential pressure transducers were used for the pressure measurements. The angle of attack was determined from an accelerometer mounted in the model.

\section{Fuselage Closure}

The three base shapes investigated on the fuselage closure-the blunt base, the blunt base with a splitter plate, and the blunt base with a vented-wall cavity-are shown in Fig. 7. All joints and openings on the body surface and in the base region were carefully sealed for each of the configurations to ensure that air did not leak from inside the fuselage. The blunt base (Figs. 2(b) and 7(a)), as indicated by the name, provided an abrupt (nearly a right angle) change in the surface contour. The top-to-bottom length of the base was $34.3 \mathrm{in}$. and the maximum width was $14.4 \mathrm{in}$. The area of the base $A_{F}$ (the area covered by the fuel dump is included) is $2.55 \mathrm{ft}^{2}$. Further details about the base are found in Powers. ${ }^{13}$

The splitter plate was attached as shown in Fig. 7(b) with a gap of 0.5 in. between the surface of the blunt base and leading edge of the splitter plate. The vertical dimension for the splitter plate was $25.0 \mathrm{in}$. and the longitudinal dimension was $14.0 \mathrm{in}$. The effective splitter plate length is $14.5 \mathrm{in}$. (sum of the gap width and the longitudinal dimension). Thus, the ratio of the effective splitter plate length (14.5 in.) to maximum base width (14.4 in.) is 1.0. The ratio used was determined from data in Nash and others. ${ }^{10}$

The vented-wall cavity modification is shown in Fig. 7(c). The sides of the vented-wall cavity were fastened to the outside of the fuselage closure. The 0.04-in. forward step discontinuity on the external wetted surface caused by the thickness of the cavity material was not significant. In general, the vented-wall cavity continued the lines of the fuselage closure. The joint between the ventedwall cavity and fuselage closure was carefully sealed to prevent air leakage. The depth of the cavity, distance from the base face to the trailing edge of the cavity, is 12.3 in. There are 11 slots on each side of the centerline (22 slots total), and the length of each slot is $9.8 \mathrm{in}$. The slot width was $0.63 \mathrm{in}$. The ratio of slot area to blunt base area $A_{v F} / A_{F}$ is 0.40 . The ratio used was determined from data in Nash. ${ }^{16}$

The base pressure for all configurations was measured by a differential pressure transducer connected to the tube manifold seen in Fig. 7(a). This tube manifold contained seven orifices positioned as indicated by the arrows. Exact locations of the seven orifices are given in Powers. ${ }^{13}$

\section{Flight Test Conditions}

The base configurations tested on the BOR for the trailing disk study were the blunt base and the fight trailing disk at $x / D$ values of 0.44 and 0.50 . The base configurations tested on the BOR for the cavity study were the extended blunt base, the solid-wall cavity, and the vented-wall cavity. The base configurations tested for the fuselage closure were the blunt base, blunt base with a splitter plate, and the blunt base with a vented-wall cavity.

Data were obtained for a 60 -sec period for each test point, beginning after the airplane had stabilized at steady-state fight 
conditions (that is, flight conditions for which the altitude and airspeed were constant). Averaged data from that period were then analyzed.

Test Mach numbers were from 0.70 to 0.95 and from 1.10 to 1.60. Data were obtained at the primary dynamic pressures of 300 and $500 \mathrm{lb} / \mathrm{ft}^{2}$. Aircraft, or free-stream, angle-of-attack values ranged from 3.9 to $6.6^{\circ}$. Aircraft, or free-stream, sideslip angles, except for a few that were near $-1.0^{\circ}$, were $\pm 0.5^{\circ}$. The rudder was in the zero, or null, position for all of the data. Data were obtained at two primary wing sweeps, 26 and $58^{\circ}$. Turbulent flow Reynolds number based on the BOR body length ranged from $1.5 \times 10^{7}$ to $2.7 \times 10^{7}$. Turbulent flow Reynolds number based on the airplane body length of $72 \mathrm{ft}$ ranged from $1.2 \times 10^{8}$ to $3.1 \times 10^{8}$.

\section{Base Drag Coefficient Analysis}

\section{Body of Revolution}

An average base drag coefficient was obtained for each base configuration by assuming that the pressure coefficient varied linearly between each pair of adjacent orifices. The drag for the blunt base, the cavity configurations, and each surface of the trailing disk configuration was determined from the following equation:

$$
D S=2 \pi q \int_{r_{\min }}^{r_{\max }} R C_{p}(R) d R
$$

where $C_{p}(R)=m R+b$. The disk surfaces were assumed to be normal to the free-stream flow from the center to the disk edge. This equation gave the base drag directly for the blunt base and the cavity configurations. However, for the trailing disk configuration, this equation gave the drag for each of the base surfaces. Thus, the base drag for the trailing disk configuration required the algebraic summation of the individual drags and was obtained by subtracting the upstream surface drag from the sum of the body base and the downstream surface drags. The base drag coefficient was then calculated using the following equation:

$$
C_{D_{b}}=D B /\left(q A_{b}\right)
$$

\section{Fuselage Closure}

The base drag coefficient $C_{D_{b}}$ for each configuration is calculated from the following equation:

$$
C_{D_{b}}=-\left(p_{b}-p_{\infty}\right) / 0.7 M_{\infty}^{2} p_{\infty}
$$

where $p_{b}$ is the base pressure, $p_{\infty}$ is the free-stream static pressure, and $M_{\infty}$ is the free-stream Mach number. Because the base pressure is measured from a seven-orifice manifold, the base drag coefficient is an average value for the base.

\section{Results and Discussion}

\section{Body of Revolution}

Base Surfaces. Surface pressure coefficients $C_{p}$ in the base region are shown in Fig. 8 as a function of ratio of radial location to the base radius $R / R B$ for flight data. Note that the center pressure
$R / R B=0$ was plotted using the symbol for the row at $16.9^{\circ}$; and, the edge pressure $R / R B=0.80$ for the flight trailing disk FLTD was plotted using the symbol for the downstream surface. The base surface pressures for the solid-wall cavity were linear with respect to radial distance (Fig. 8(a)). This linear relationship was typical for all the cavity and blunt base configurations.

The pressure coefficients $C_{p}$ as a function of $R / R B$ are shown in Figs. 8(b) and $8(\mathrm{c})$ for the FLTD, $x / D=0.44$. The linear relationship for the downstream disk surface and the nonlinear relationship for the BOR and upstream disk surfaces were typical for the trailing disk configurations. The pressure coefficients in the cavity formed by the BOR base and the upstream surface of the trailing disk are almost the same. Thus, the net drag from this region of the base is close to zero. The pressure coefficients on the downstream surface of the trailing disk and the remaining surface area (the annular area beyond or outside the shadow of the disk) of the BOR base dominate the calculation for base drag coefficient. This was true for all the trailing disk configurations. Surface pressure coefficients for all the Mach numbers and configurations of the flight and wind-tunnel study are found in Powers and others. ${ }^{12}$

Flight Base Drag Coefficient. The incremental differences between the flight base drag coefficient for the blunt base and for the trailing disk and cavity configurations are shown in Fig. 9. The base drag coefficients for the blunt and the extended blunt bases were the same; therefore, no distinction is made between them. Both trailing disk configurations have a significant decrease in base drag coefficient for the Mach number range of 0.70 to 0.93. The decreases range from 0.08 to 0.07 for the $x / D=0.50$ configuration and from 0.08 to 0.06 for the $x / D=0.44$ configuration as Mach number increases from 0.70 to 0.93 . The decrease in base drag coefficient with the cavity configurations (0.01 maximum) is not considered significant. For these values of $d / D$ and $A_{v B} / A_{B}$, the incompressible flow data in Mair ${ }^{5}$ had decreases in base drag coefficient of 0.03 for the solid-wall cavity and 0.07 for the vented-wall cavity. Percentage changes for the base drag reduction are not given for the flight data because of the low values for the blunt base drag coefficient.

Some supersonic data (not shown here) were obtained during the flight trailing disk study. ${ }^{12}$ The trailing disk configurations caused a base drag penalty at the lower supersonic speeds; however, the limited data indicate that this penalty decreases rapidly as Mach number increases to 1.4 and the penalty approaches zero as Mach number increases from 1.4 to 1.6 .

Effect of Separation Distance. The longer stem of the wind-tunnel trailing disk (WTD) allowed a larger range in $x / D$ values than was possible with the FLTD. Thus, the effect of $x / D$ on the base drag coefficient (from the pressure data) and on the total drag coefficient (from the force-balance data) was obtained from the wind-tunnel test. The difference between the base drag coefficient for the blunt base BOR and the BOR with either the WTD or the FLTD is shown in Fig. 10(a). The difference between the total drag coefficient for the blunt base BOR and the BOR with either the WTD or the FLTD is shown in Fig. 10(b). The maximum decrease, or improvement, from the blunt base configuration in base drag and total drag coefficients ranged from 0.08 to 0.05 as 
Mach number increased from 0.30 to 0.82 . These decreases corresponded to decreases from 52 to 29 percent for the base drag coefficient and from 31 to 18 percent for the total drag coefficient as Mach number increased. The wind-tunnel data for $\alpha \simeq 3^{\circ}$ were more limited in $x / D$ and Mach number ranges. These data (not shown) had similar reductions in base and total drag coefficients.

Flight and Wind-Tunnel Data Comparisons. Comparisons between the increments of the trailing disk configurations for the flight and wind-tunnel data of Powers and others ${ }^{12}$ and their respective blunt bases are shown in Fig. 11. These differences are presented as a function of $x / D$ in Fig. 11(a) and as a function of Mach number in Fig. 11(b). Only values of $x / D$ near those of the flight data are shown. The data for the trailing disk in flight have a larger decrease in the base drag coefficient than do the trailing disk data from the wind-tunnel study. However, both the data from the flight experiment and the data from the full-scale wind-tunnel model experiment demonstrate the significant base drag reduction (and also total BOR drag reduction) capability of the trailing disk concept to 0.93 Mach number. The larger decrease observed in base drag coefficient for the flight data is probably caused by the different mounting structures (Figs. 2(a) and 6). In Powers and others, ${ }^{12}$ these different mounting structures are seen to cause a substantial difference in the pressure field of the BOR.

The maximum differences in the base drag and total drag coefficients for the trailing disk data (irrespective of $x / D$ ) are presented in Fig. 12 as a function of Mach number. Data shown for zero degree angle of attack, approximately 0.1 Mach number, and Reynolds numbers from $0.4 \times 10^{6}$ to $1.5 \times 10^{6}$ are from Mair, ${ }^{3}$ Goodyer, ${ }^{4}$ and Little and Whipkey. ${ }^{17}$ The trailing disk data of the present study, in general, have larger reductions in the base drag and total drag coefficients than do the lower Reynolds number, incompressible data of the previous studies. ${ }^{3,4,17}$ Even though the levels are different, the data of the previous studies and of the present study all show substantial reductions in both the base drag and total drag coefficients. The differences between the present study and previous studies could be owing to a number of factors. Besides differences in the mounting structures, there were also significant differences in Mach numbers and Reynolds numbers.

\section{Fuselage Closure}

Because of the nearness of the engine exhaust nozzles to the fuselage closure, it is desirable to establish that the flow from the exhaust nozzle is similar for all the configurations. The engine parameter used to compare the similarity of the engine conditions is the nozzle pressure ratio, the ratio of turbine discharge total pressure to free-stream pressure $p_{T_{7}} / p_{\infty}$. In Fig. 13, $p_{T,} / p_{\infty}$ is shown as a function of Mach number for all the configurations. The agreement between the average values for the blunt base and the modifications is good. These comparisons provide assurance that, at a given Mach number, any differences observed between the base drag coefficients for the blunt base and the modifications are caused by the modification, and that these differences are not significantly influenced by propulsion factors.

The increment in base drag coefficient between the blunt base and the blunt base with either a splitter plate or vented-wall cavity $\left(A_{v F} / A_{F}=0.40\right)$ is shown as a function of Mach number in Fig. 14. Averaged base drag coefficients (given in Powers ${ }^{13}$ ) were used in the calculations. The data for the blunt base is the baseline, or reference, condition. For Mach numbers $<1$, the increment ranges between \pm 0.01 . These increments are not considered significant. For Mach numbers $>1$, the vented-wall cavity has significant reductions in base drag coefficient. The reductions range from 0.05 to 0.07 . The 0.02 reduction in base drag coefficient for the splitter plate at 1.51 is offset by the small increases (< $-0.01)$ at 1.10 and 1.31 Mach numbers.

The possible effects of angle of attack or Reynolds number on the base drag coefficient at a given Mach number were investigated for each of the base configurations, but no apparent relationship was detected. The lack of a relationship was not unexpected because turbulent flow begins far upstream of the base region, and turbulent flow is relatively insensitive to the modest excursions in angle of attack and Reynolds number experienced during this study. Also, the base drag coefficient, at a given Mach number, was not found to be influenced by either the different wing sweeps or the different dynamic pressures.

To better relate the increments in base drag coefficient to a reduction in base drag, assume a flight condition having a free-stream dynamic pressure of $500 \mathrm{lb} / \mathrm{ft}^{2}$. For this dynamic pressure, altitude varies from 31,400 to $44,300 \mathrm{ft}$ as Mach number increases from 1.10 to 1.51 . Then, for the $2.55 \mathrm{ft}^{2}$ base area of this study, each 0.01 increment in base drag coefficient means a $12.75 \mathrm{lb}$ reduction in base drag. The base drag reductions obtained for the vented-wall cavity for each supersonic test Mach number are presented in the following table:

\begin{tabular}{cccc}
\hline \hline Mach & $\begin{array}{c}\text { Increment, } \\
\Delta C_{D_{\mathrm{b}}}\end{array}$ & $\begin{array}{c}\text { Base drag, } \\
\text { lb }\end{array}$ & $\begin{array}{c}\text { Reduction, } \\
\text { percent }\end{array}$ \\
\hline 1.10 & 0.07 & 89 & - \\
1.31 & 0.05 & 64 & 27 \\
1.51 & 0.06 & 76 & 24 \\
\hline
\end{tabular}

Because the base drag coefficient for the blunt base is near zero for the 1.10 Mach number data, the percentage change is not calculated.

These reductions in base drag are translated to a change in total aircraft drag for the 1.51 Mach number data. A $500 \mathrm{lb} / \mathrm{ft}^{2}$ dynamic pressure is again assumed (corresponds to an altitude of $44,300 \mathrm{ft}$ ). Then for an angle of attack of $5.4^{\circ}$, a total aircraft drag coefficient of 0.0490 , based on a wing area of $604 \mathrm{ft}^{2}$, was obtained for the F-111 TACT from Cooper and others. ${ }^{14}$ Using the F-111 TACT wing area of $604 \mathrm{ft}^{2}$, the 76-lb base drag reduction resulted in 0.5 percent reduction in total aircraft drag or a 0.5 percent increase in range.

\section{Conclusions}

The effectiveness of a trailing disk, solid-wall cavity or ventedwall cavity in reducing the base drag of a large body of revolution, with 8-in. diameter, was studied. Also studied was the effectiveness of a vented-wall cavity and a splitter plate in reducing the base drag of a quasi-two-dimensional body (F-111 fuselage closure) in the presence of jet exhaust. The data were analyzed using the blunt 
base for a reference, or baseline, configuration. The analysis led to the following conclusions:

1. For the body of revolution experiment, data from both the flight and full-scale wind-tunnel model tests demonstrate the significant base drag reduction (and also total drag reduction) capability of the trailing disk concept to 0.93 Mach number. The maximum decreases in base drag coefficient for the flight trailing disk data ranged from 0.08 to 0.07 as Mach number increased from 0.70 to 0.93 . For the trailing disk data from the full-scale wind-tunnel model experiment, the maximum decrease in base drag and total drag coefficients ranged from 0.08 to 0.05 as Mach increased from 0.30 to 0.82 . These decreases corresponded to percent decreases of from 52 to 29 percent for the base drag coefficient and from 31 to 18 percent for the total drag coefficient as Mach number increased. For the cavity data of the flight experiment, the decrease in base drag coefficient is not considered significant.

2. For the F-111 fuselage closure experiment, the reduction in base drag coefficient provided by the vented-wall cavity base ranged from 0.07 to 0.05 as Mach number increased from 1.10 to 1.51 . Base drag reductions of 89,64 , and $76 \mathrm{lb}$ were oblained for Mach numbers of 1.10, 1.31, and 1.51, respectively, for a dynamic pressure of $500 \mathrm{lb} / \mathrm{ft}^{2}$. The increments in base drag coefficient at Mach numbers of 1.31 and 1.51 result in base drag reductions of 27 and 24 percent, respectively, when compared to the blunt base drag. The splitter plate is not considered effective in reducing base drag at any Mach numbers tested.

\section{References}

'Ringleb, F.O.: Separation Control by Trapped Vortices, in Boundary Layer and Flow Control, G.V. Lachmann, ed. Pergamon Press, 1961, pp. 265-294.

${ }^{2}$ Migay, V.K. (Translation Div., Foreign Technology Div., WrightPatterson AFB): Investigating Finned Diffusers. Available from DDC as AD-402582, 1963. (Primary source-Teploenergetika, no. 10 , Oct. 1962, in Russian.)

${ }^{3}$ Mair, W.A.: The Effect of a Rear-Mounted Disc on the Drag of a Blunt-Based Body of Revolution. Aeronaut. Quart., vol. 16, Nov. 1965, pp. 350-360.

${ }^{4}$ Goodyer, M.J.: Some Experimental Investigations Into the Drag Effects of Modifications to the Blunt Base of a Body of Revolution. Institute of Sound and Vibration Research, Southhampton University, United Kingdom, report no. 150, July 1966.
${ }^{5}$ Mair, W.A.: Drag-Reducing Techniques for Axi-Symmetric Bluff Bodies, in Aerodynamic Drag Mechanisms of Bluff Bodies and Road Vehicles, Gino Sovran, Thomas Morel, and W.T. Mason, Jr., eds. Plenum Press, 1978, pp. 161-187.

${ }^{6}$ Hoener, S.F.: Fluid-Dynamic Drag. Second ed. Published by the author (148 Busteed Drive, Midland Park, New Jersey), 1965.

${ }^{7}$ Roshko, Anatol: On the Drag and Shedding Frequency of Two-Dimensional Bluff Bodies. NACA TN-3169, 1954.

${ }^{8}$ Bearman, P.W.: Investigation of the Flow Behind a TwoDimensional Model With a Blunt Trailing Edge and Fitted With Splitter Plates. J. Fluid Mech., vol. 21, part 2, Feb. 1965, pp. 241 256.

${ }^{9}$ Saltzman, E.J.; and Hintz, John: Flight Evaluation of SplitterPlate Effectiveness in Reducing Base Drag at Mach Numbers From 0.65 to 0.90. NASA TM X-1376, 1967.

${ }^{10}$ Nash, J.F.; Quincey, V.G.; and Callinan, J.: Experiments on Two-Dimensional Base Flow at Subsonic and Transonic Speeds. Ministry of Aviation of United Kingdom, Aeronautical Research Council, Reports and Memoranda no. 3427, 1966.

${ }^{11}$ Painter, W.D.; and Caw, L.J.: Design and Physical Characteristics of the Transonic Aircraft Technology (TACT) Research Aircraft. NASA TM-56048, 1979.

${ }^{12}$ Powers, S.G.; Huffman, J.K.; and Fox, C.H., Jr.: Flight and Wind-Tunnel Measurements Showing Base Drag Reduction Provided by a Trailing Disk for High Reynolds Number Turbulent Flow for Subsonic and Transonic Mach Numbers. NASA TP-2638, 1986.

${ }^{13}$ Powers, S.G.: Influence of Base Modifications on In-Flight Base Drag in the Presence of Jet Exhaust for Mach Numbers from 0.7 to 1.5. NASA TP-2802, 1988.

${ }^{14}$ Cooper, J.M., Jr.; Hughes, D.L.; and Rawlings, Kenneth, III: Transonic Aircraft Technology-Flight-Derived Lift and Drag Characteristics, Volume I of II. Air Force Flight Test Center, Edwards Air Force Base, AFFTC-TR-77-12, 1977.

${ }^{15}$ Fox, C.H., Jr.; and Huffman, J.K.: Calibration and Test Capabilities of the Langley 7- by 10-Foot High Speed Tunnel. NASA TM X-74027, 1977.

${ }^{16}$ Nash, J.F.: A Discussion of Two-Dimensional Turbulent Base Flows. National Physical Laboratory, United Kingdom, NPL Aero report 1162, 1965.

${ }^{17}$ Little, B.H., Jr.; and Whipkey, R.R.: Locked Vortex Afterbodies. AIAA-78-1179. Presented to the 11 th Fluid and Plasma Dynamics Conference, Seattle, Washington, July 10-11, 1978. 
ORIGINAL PAGE IS

OF POOR ?:UITY

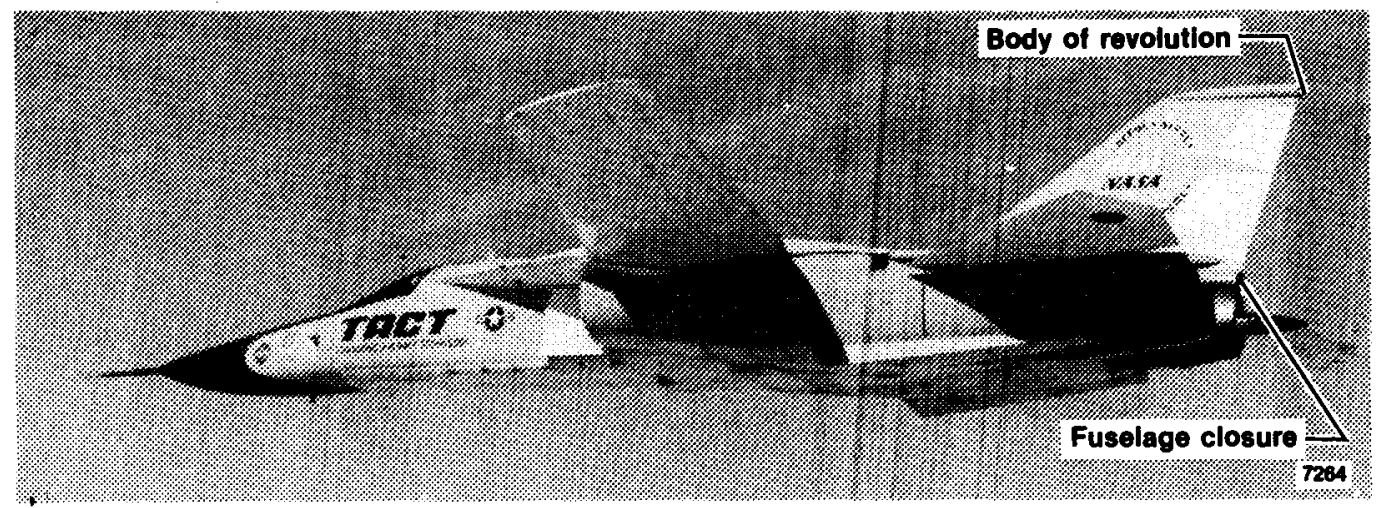

Fig. 1 F-111 TACT airplane in flight.

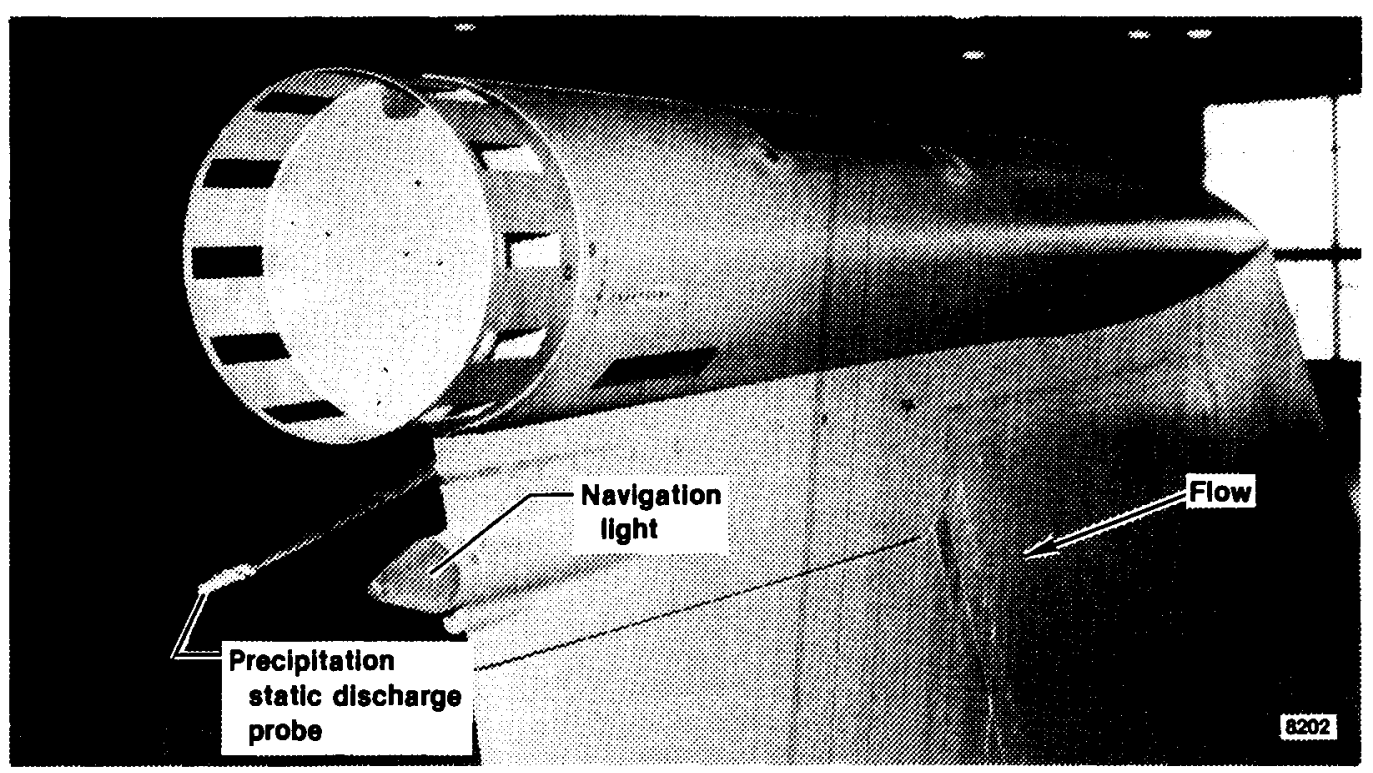

(a) Three-quarter rear view of BOR and portion of vertical tail. (Vented-wall cavity base is installed.)

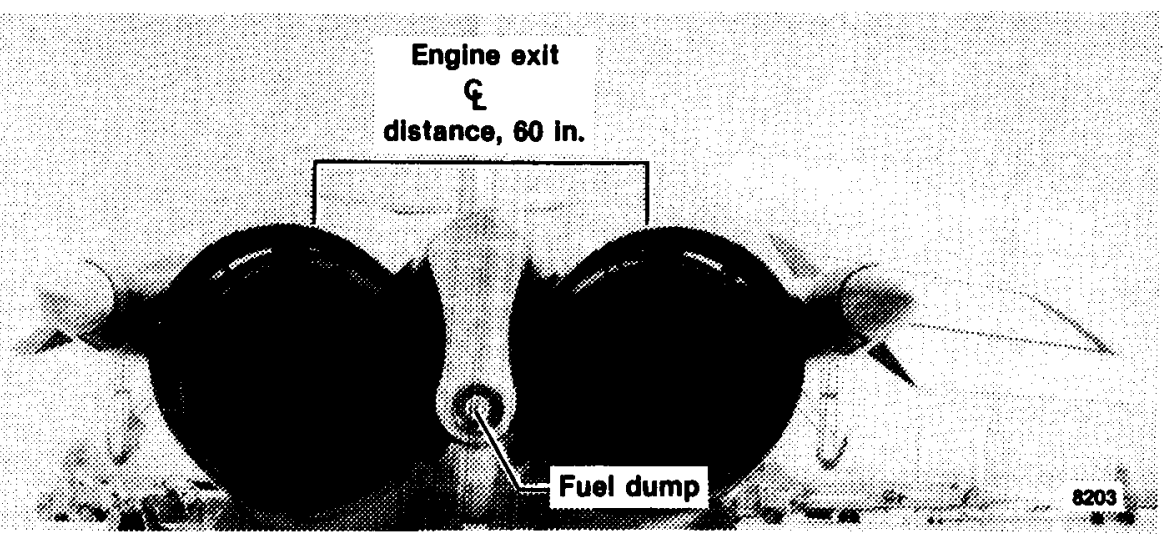

(b) Rear view of the F-111 aircraft. Nozzles are fully open.

Fig. 2 Close-up of experiment locations. 


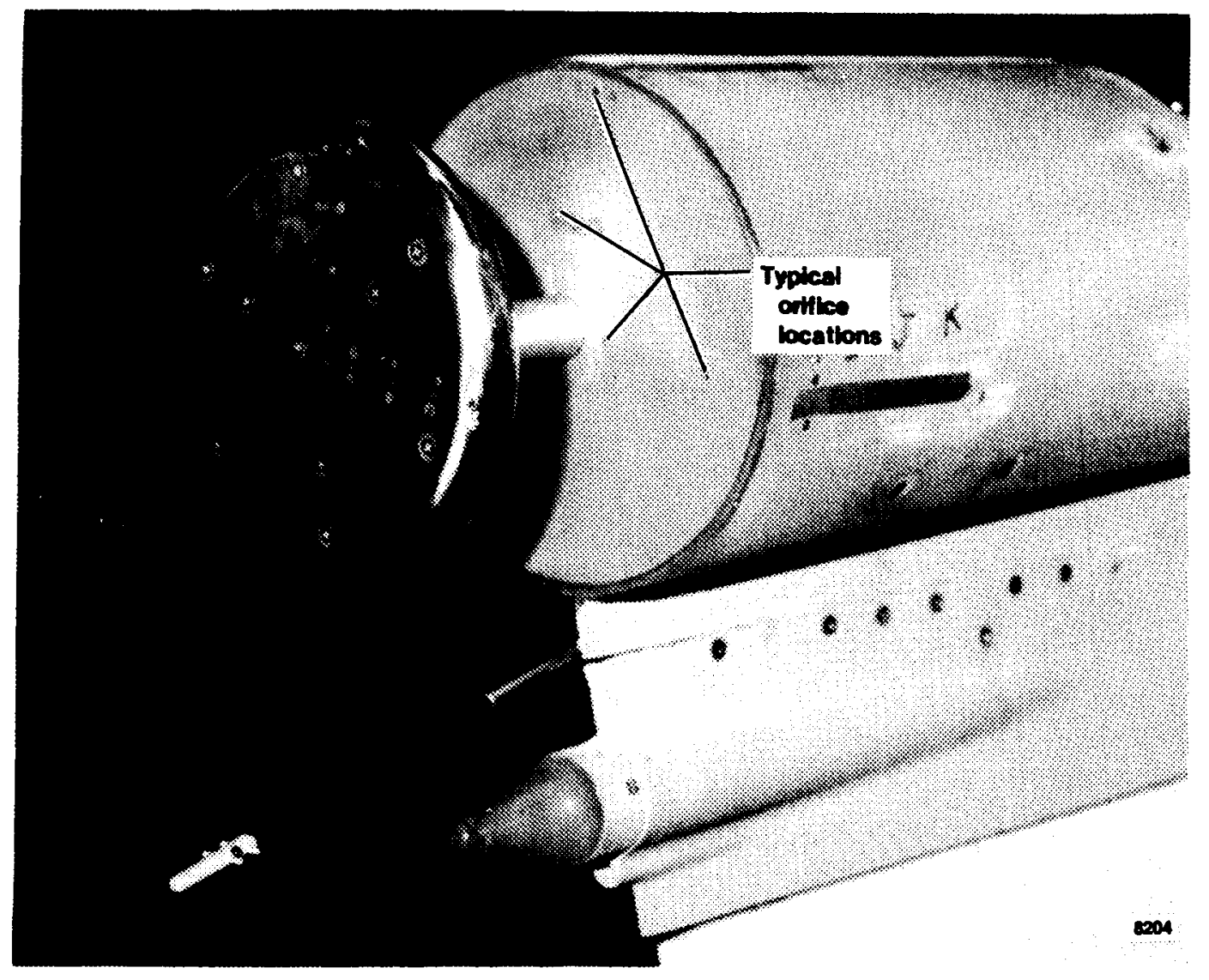

(a) Installed on BOR.

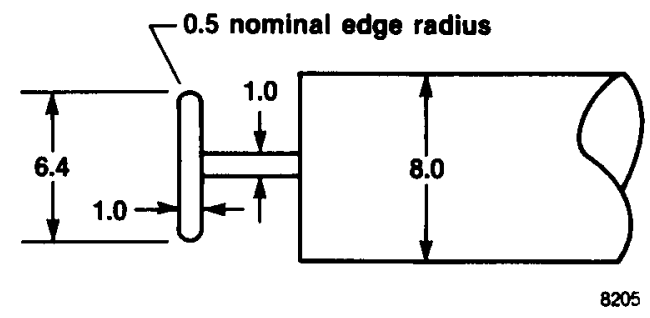

(b) Dimensions, in.

Fig. 3 Trailing disk tested on BOR, flight study. 


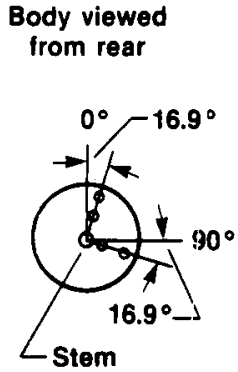

Body base

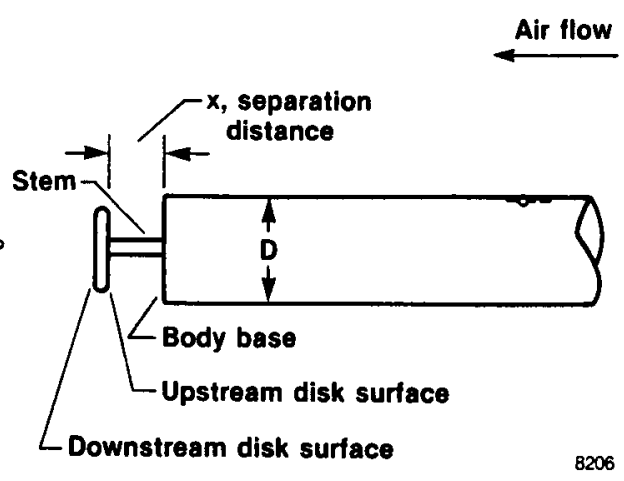

Fig. 4. Nomenclature and angular orientation $(\theta)$ of pressure orifice rows for trailing disk study.

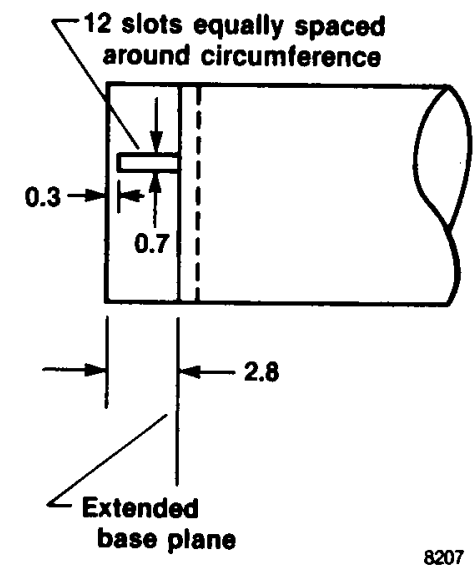

Fig. 5 Vented-wall cavity dimensions, in. Dashed line indicates original base plane of BOR.

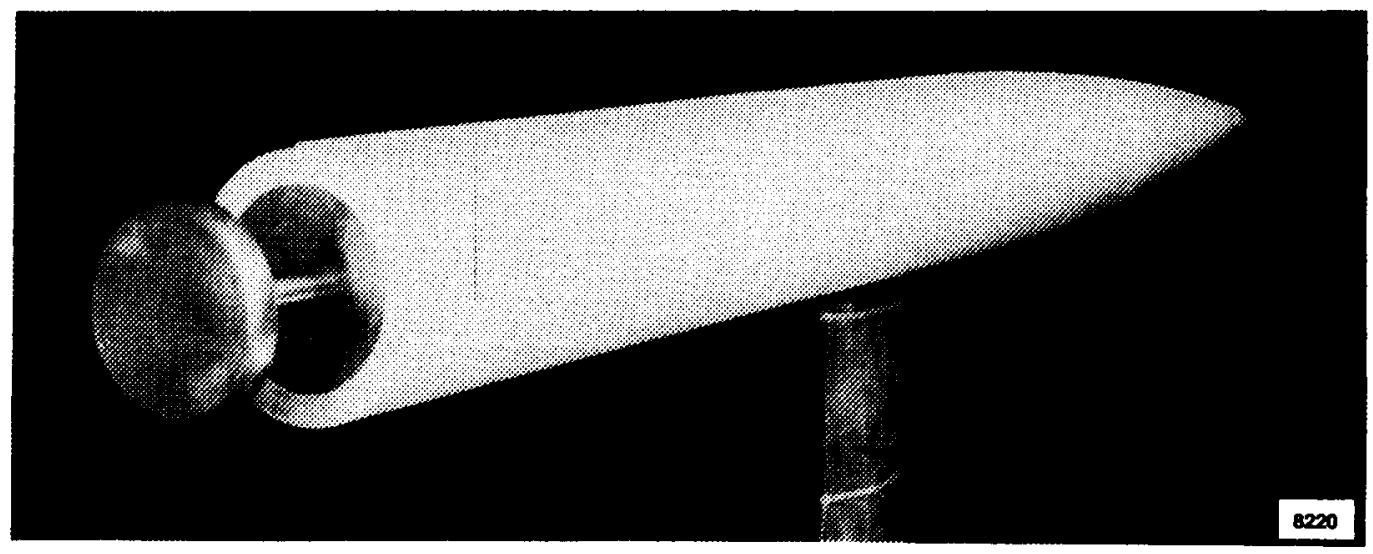

L 78-7515

Fig. 6 Wind-tunnel BOR installed in Langley high-speed 7- by 10-ft tunnel; wind-tunnel trailing disk installed. 


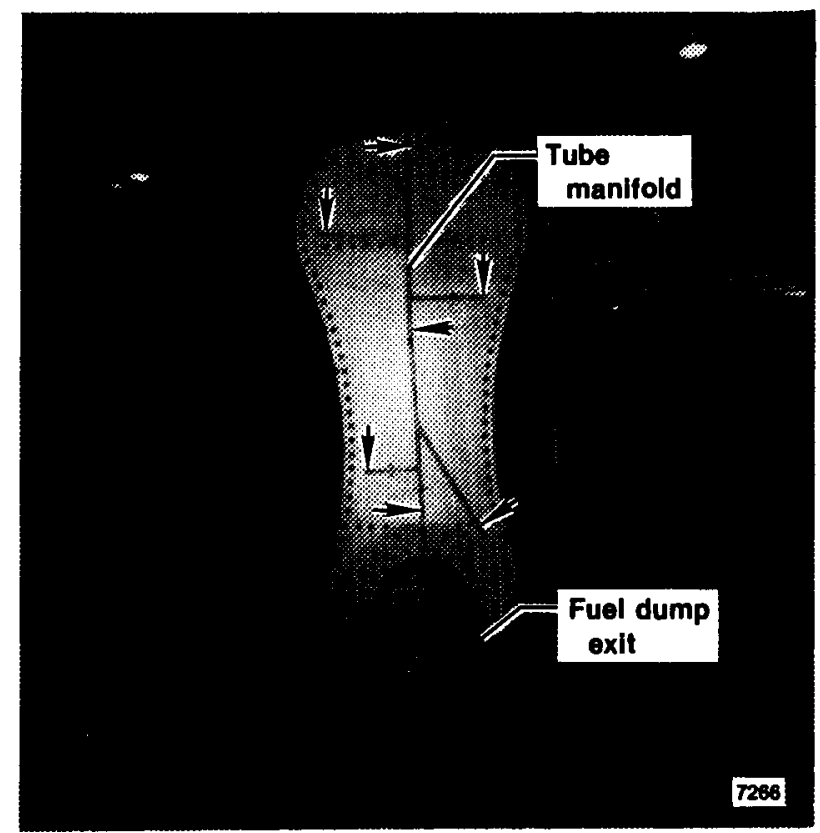

(a) Blunt base. Arrows indicate orifice locations in tube manifold.

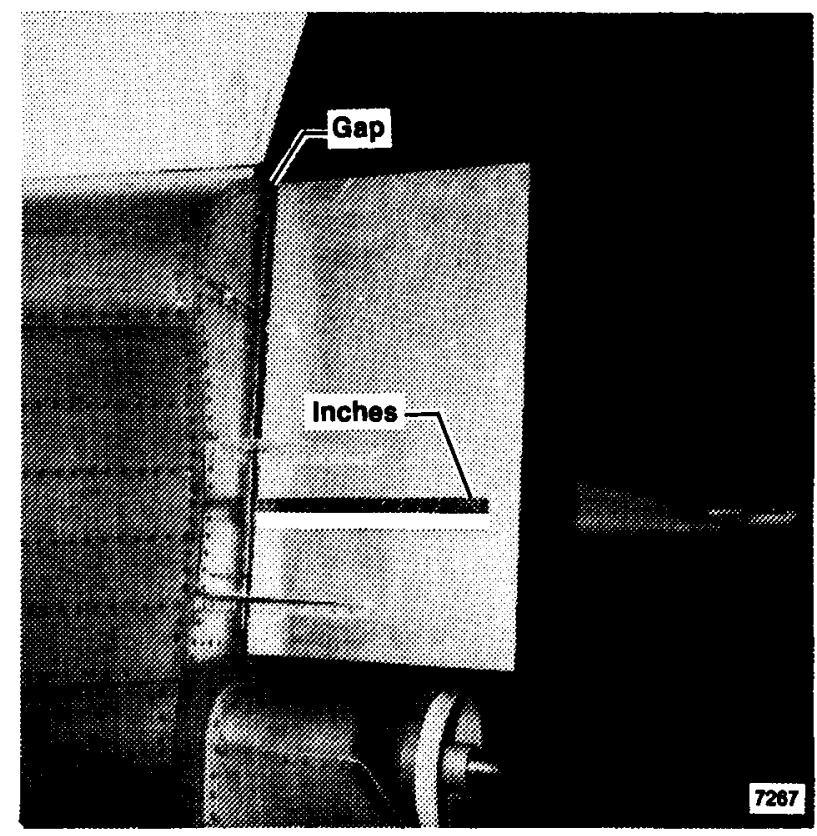

(b) Blunt base with splitter plate.

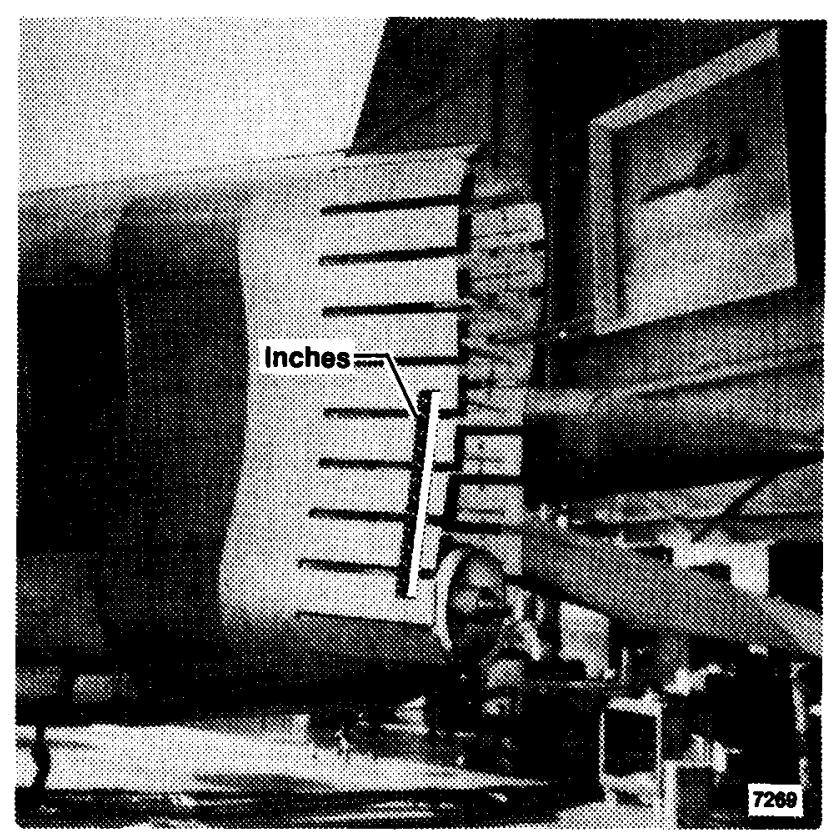

(c) Blunt base with vented-wall cavity.

Fig. 7 Fuselage closure base configurations. 


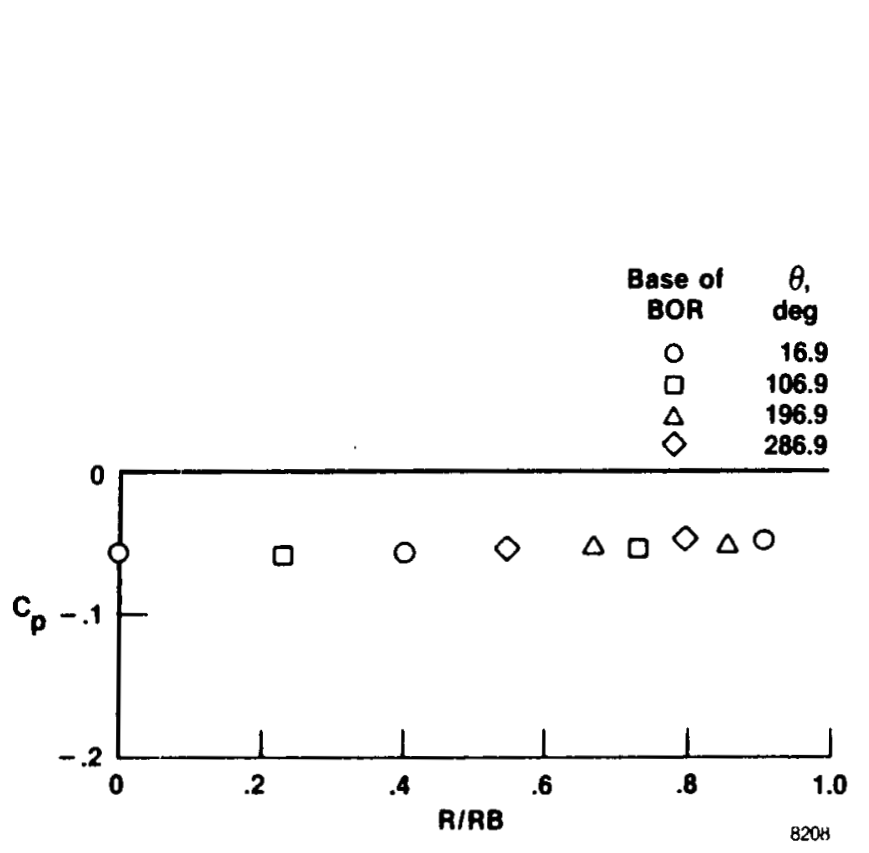

(a) Solid-wall cavity, $M=0.70$.
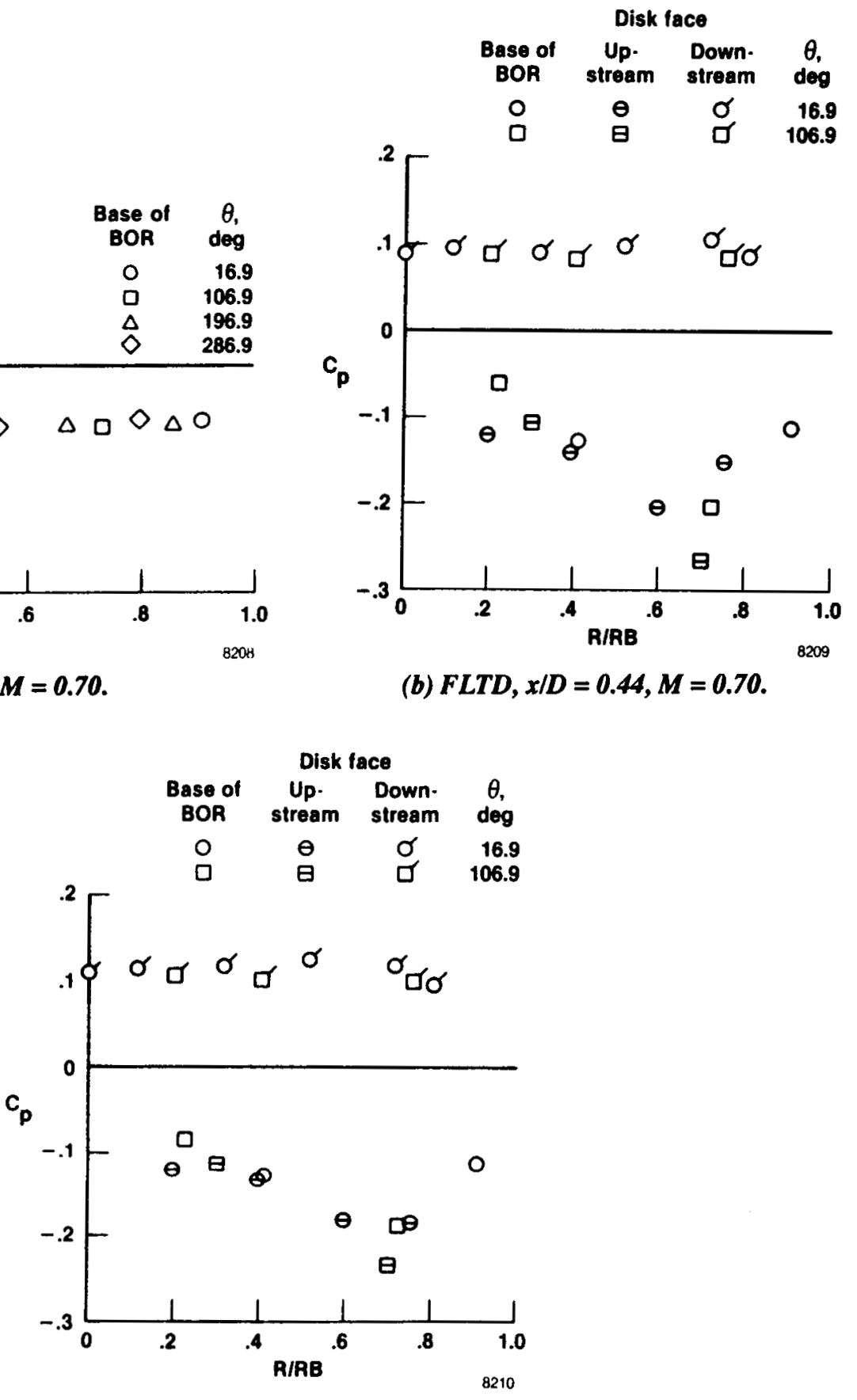

(c) FLTD, $x / D=0.44, M=0.93$.

Fig. 8 Pressure coefficient in base region as a function of radial distance for BOR base configurations, flight data. 


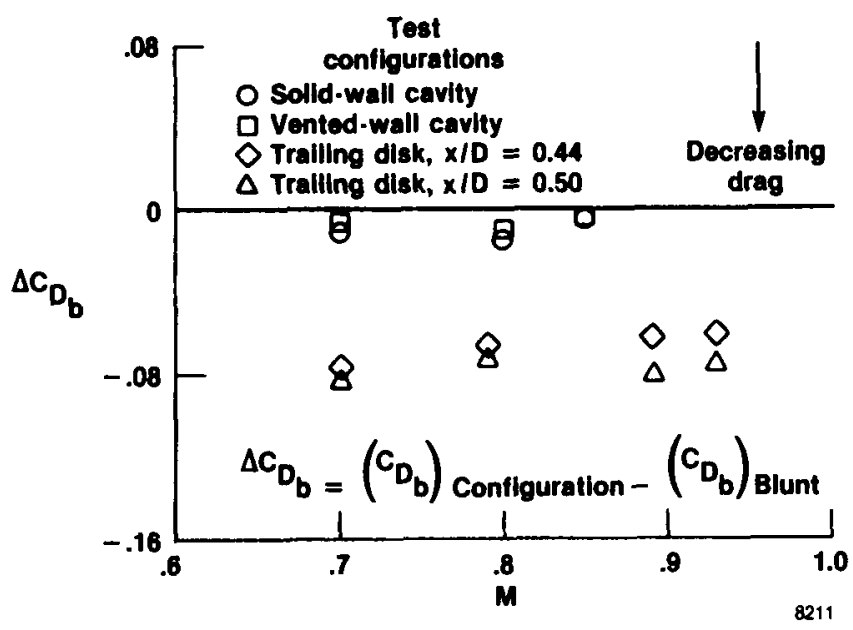

Fig. 9 Incremental difference in BOR base drag coeffcient between given configuration and blunt base, flight data.

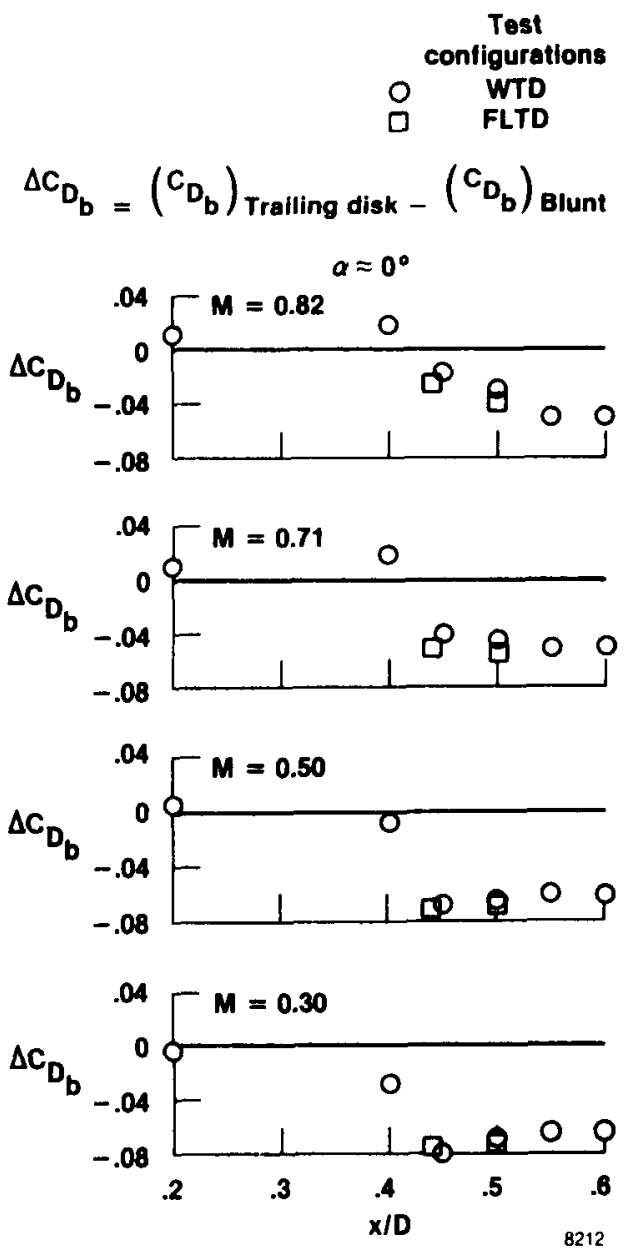

(a) Increment in base drag coefficient. (Negative means base drag was less than blunt base drag.)

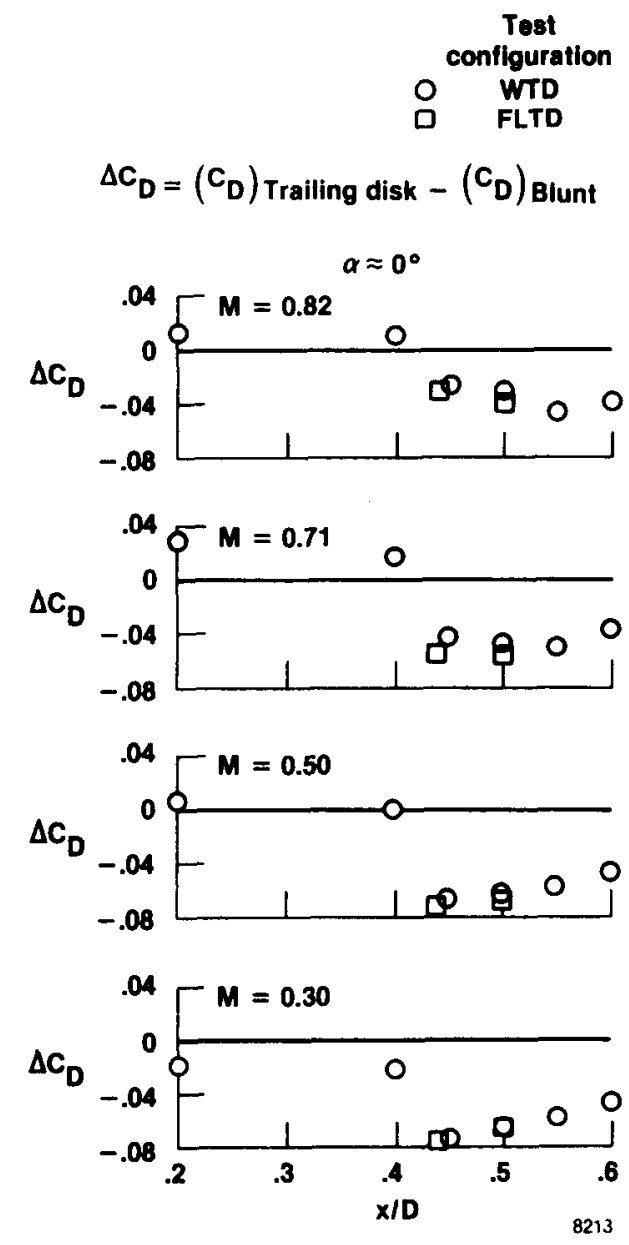

(b) Increment in total drag coefficient. (Neg* ative means $B O R$ with trailing disk had less total drag than BOR with blunt base.)

Fig. 10 Drag coefficient increment between given trailing disk configuration and blunt base as a function of $x / D$, wind-tunnel BOR study. 


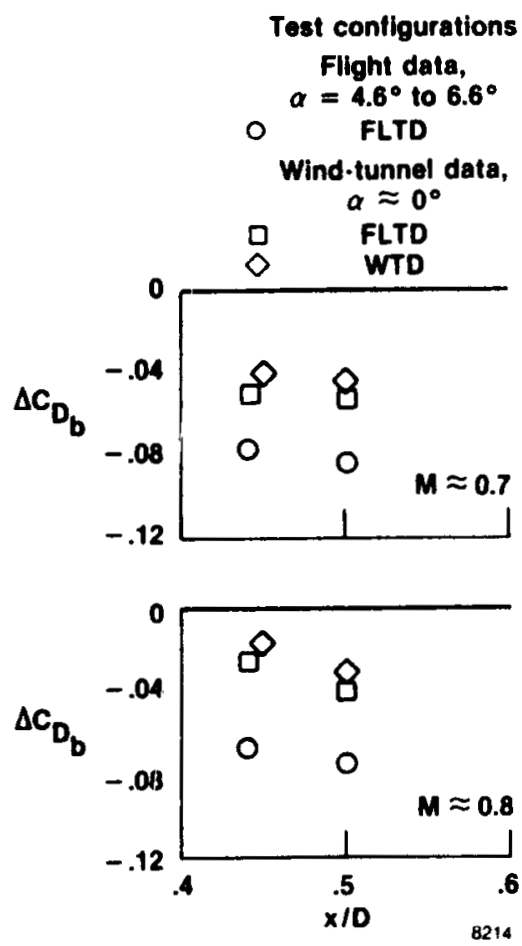

(a) $\Delta C_{D_{b}}$ vs. $x / D$.
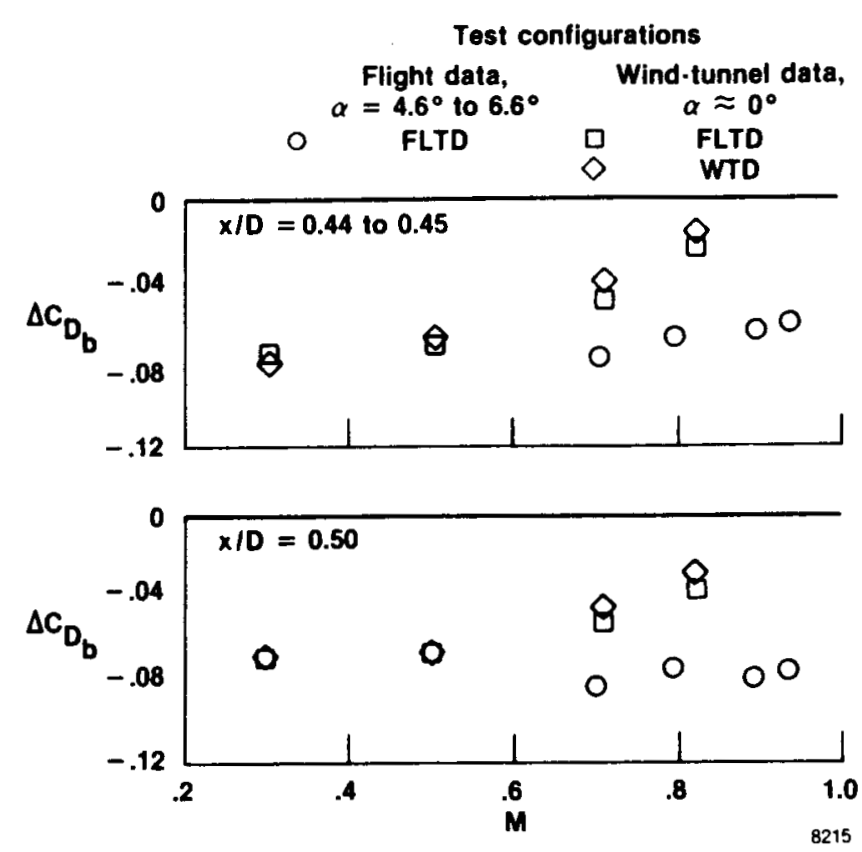

(b) $\Delta C_{D_{\mathbf{b}}}$ vs. $M$.

Fig. 11 Comparison of flight and wind-tunnel BOR base drag coefficients with the trailing disk, $x / D=0.44$ to 0.50 .

(Flight data $\alpha$ is aircraft angle of attack; negative means a drag decrease.)

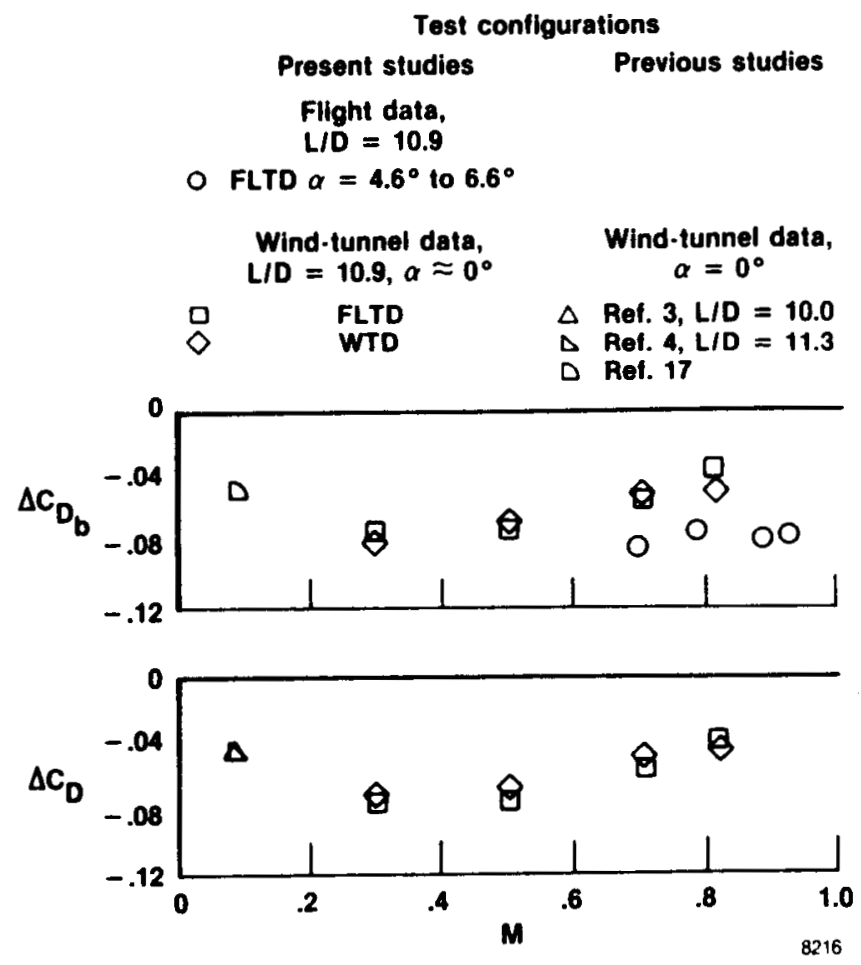

Fig. 12 Comparison of maximum drag coefficient reduction for flight and wind-tunnel BOR data of present study and previous wind-tunnel studies. (Flight data $\alpha$ is aircraft angle of attack; negative means a drag decrease.) 


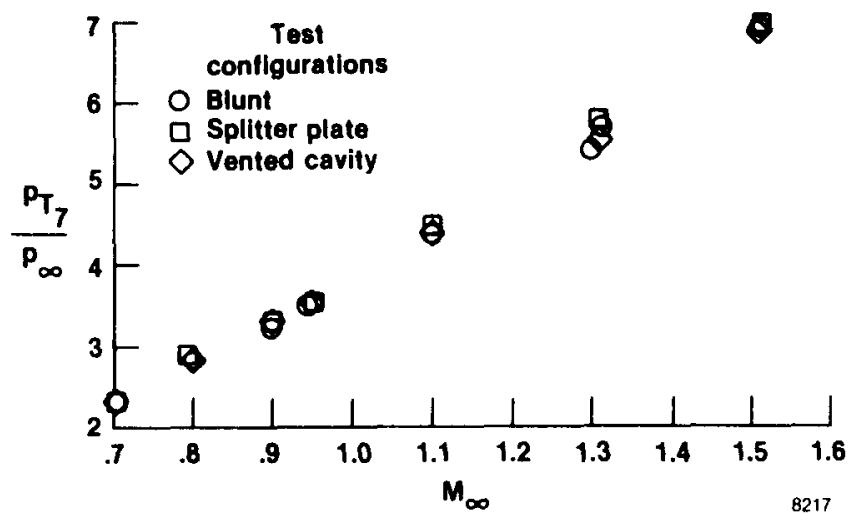

Fig. 13 Comparison of average ratio of turbine discharge total pressure to free-stream pressure for the different configurations, flight data.

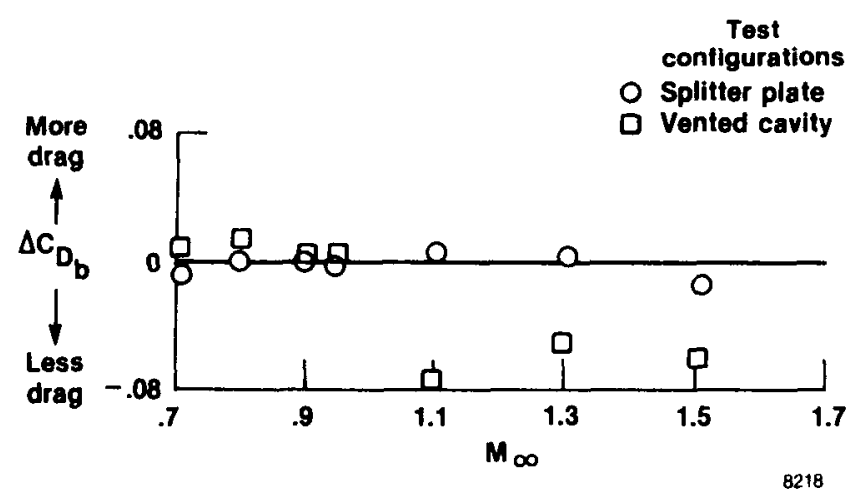

Fig. 14 Incremental difference in base drag coefficient between blunt base and other configurations for fuselage closure experiment, fight data. 


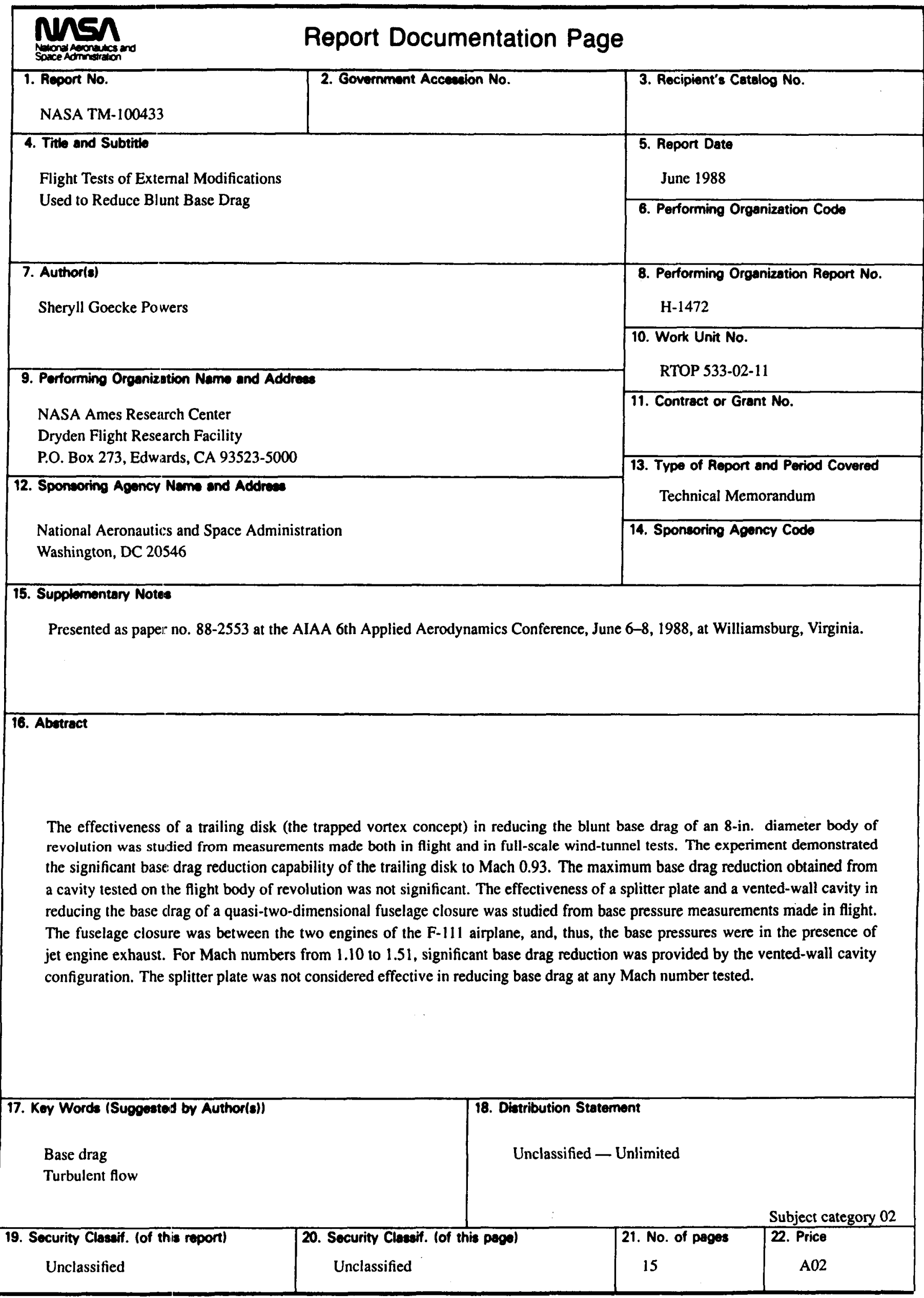

